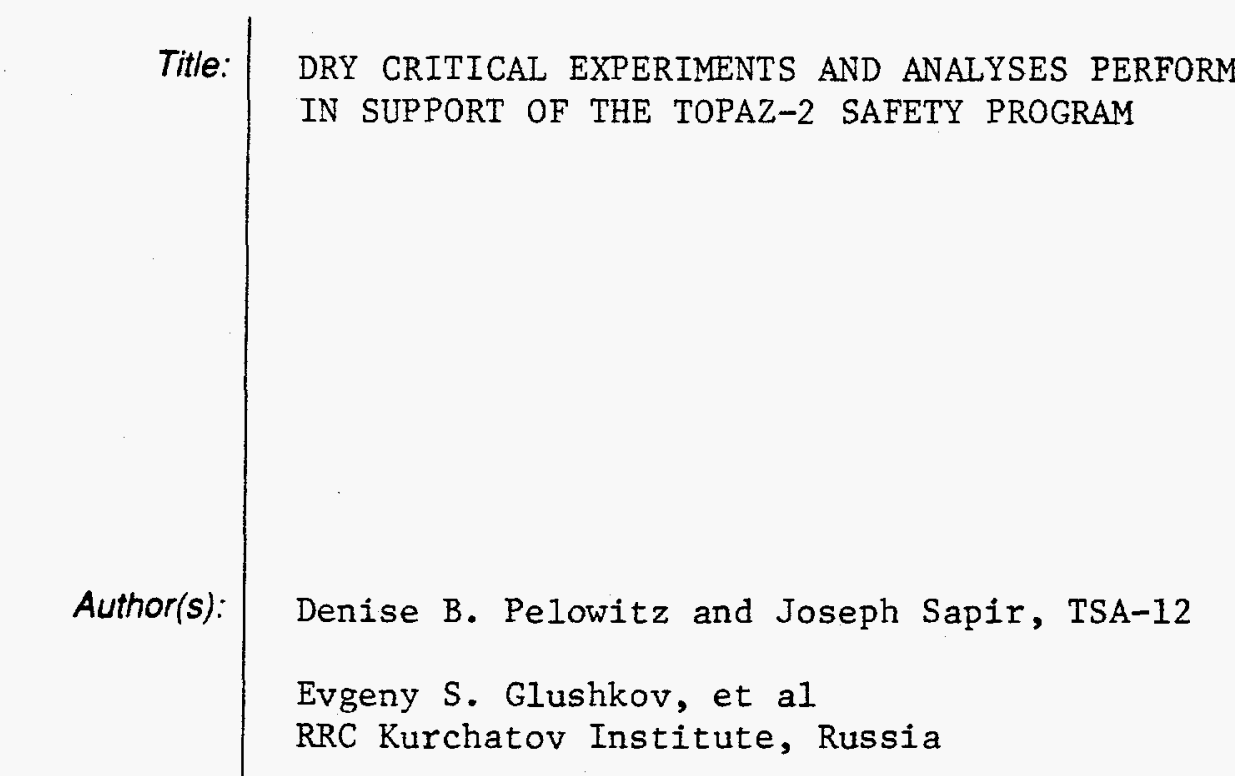

Submitted to:

Twelfth Symposium

on Space Nuclear Power Propulsion

Albuquerque, NM

January 8-12, 1995

DISTRIBUTION OF THIS DOCUMENT IS UNLIMITED

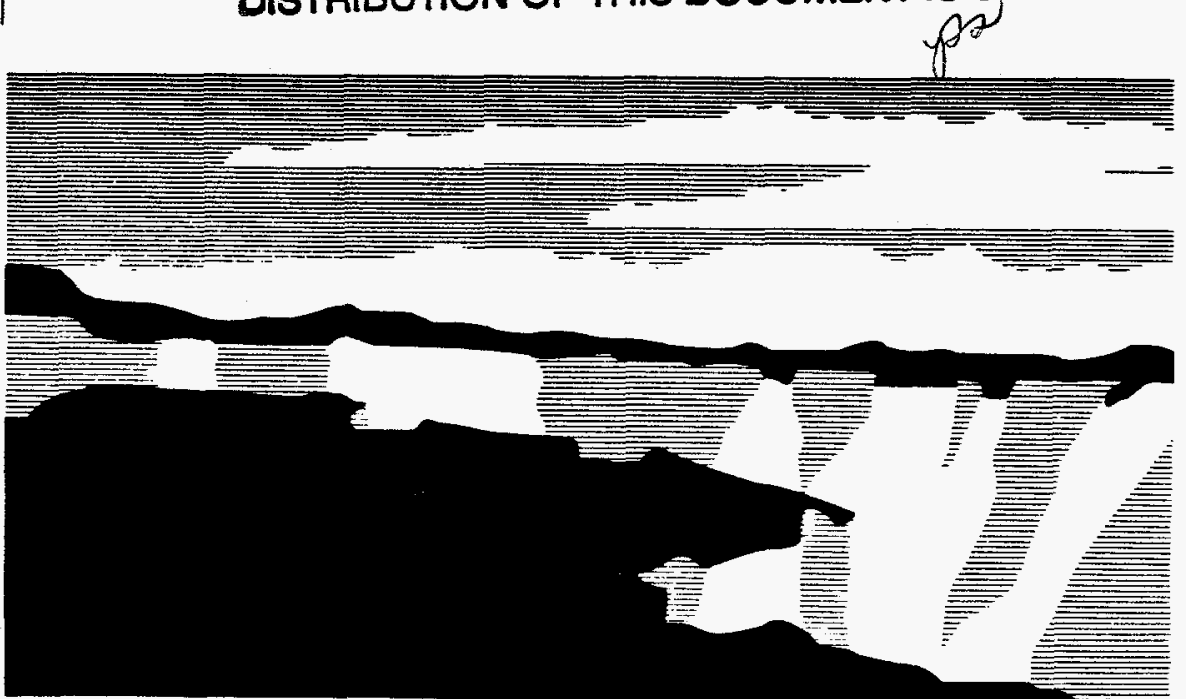

Los Alamos National Laboratory, an affirmative action/equal opportunity empldyer, is operated by the University of California tor the U.S. Department of Eneroy under contract W-7405-ENG-36. By acceptance of this article, the publisher recognizes that the U.S. Government retains a nonexclusive, royalty-free license to publish or reproduce the published form of this contribution, or to allow others to do so, for U.S. Government purposes. The Los Alamos National Laboratory requests that the publisher identily this article as work performed under the auspices of the U.S. Department of Energy. 


\section{DISCLAIMER}

Portions of this document may be illegible in electronic image products. Images are produced from the best available original document. 


\title{
DRY CRITICAL EXPERIMENTS AND ANALYSES PERFORMED IN SUPPORT OF THE TOPAZ-2 SAFETY PROGRAM
}

\author{
Denise B. Pelowitz and Joseph Sapir \\ Reactor Design and Analysis Group \\ TSA-12, MS K551 \\ Los Alamos National Laboratory \\ Los Alamos, NM 87545 \\ (505) 667-0912
}

\author{
Evgeny S. Glushkov, Nikolai N. Ponomarev-Stepnoi, \\ Vladimir G. Bubelev, George B. Kompanietz, \\ Aleksei M. Krutov, Dmitry N. Polyakov, \\ and Viacheslav A. Lobynstev \\ RRC Kurchatov Institute \\ Moscow 123182 Russia
}

\begin{abstract}
In December 1991, the Strategic Defense Initiative Organization decided to investigate the possibility of launching a Russian Topaz-2 space nuclear power system. Functional safety requirements developed for the Topaz mission mandated that the reactor remain subcritical when flooded and immersed in water. Initial experiments and analyses performed in Russia and the United States indicated that the reactor could potentially become supercritical in several water- or sand-immersion scenarios. Consequently, a series of critical experiments was performed on the Narciss M-II facility at the Kurchatov Institute to measure the reactivity effects of water and sand immersion, to quantify the effectiveness of reactor modifications proposed to preclude criticality, and to benchmark the calculational methods and nuclear data used in the Topaz-2 safety analyses. In this paper we describe the Narciss M-II experimental configurations along with the associated calculational models and methods. We also present and compare the measured and calculated results for the dry experimental configurations.
\end{abstract}

\section{INTRODUCTION}

In December 1991, the Strategic Defense Initiative Organization (SDIO) decided to investigate the possibility of launching a Russian Topaz-2 space nuclear power system. A joint effort between U.S. and Russian scientists was initiated to develop the information necessary to assess the safety of such a mission. Functional safety requirements developed for the Topaz mission mandated that the reactor remain subcritical when flooded and immersed in water. Initial experiments and analyses performed in Russia and the U.S. indicated that the reactor could potentially become supercritical in several water- and sand-immersion accident scenarios. The U.S. and Russian teams jointly investigated potential reactor modifications that would assure that the reactor would remain subcritical before the planned reactor startup. Two concepts were identified and studied: (1) insertion of a selected number of poison rods inside the core, and (2) removal of a selected number of fuel elements from the core until a nuclear-safe orbit is established. The "fuel-out anticriticality device" was chosen as the baseline hardware modification to prevent preorbital criticality.

Consequently, a series of critical experiments was performed on the Narciss M-II facility at the Kurchatov Institute to measure the reactivity effects of water and sand immersion, and to quantify the effectiveness of reactor modifications proposed to preclude criticality. Although the Narciss M-II configuration is a fairly faithful simulation of the expected flight reactor, the experimental results cannot be directly used to predict the behavior of the flight system, but are most appropriately used to benchmark the nuclear data and neutron-transport methods used by the U.S. and Russian teams and to determine uncertainty bounds of the flight-unit safety calculations.

In this paper we describe the Narciss M-II configuration, review the critical experiments performed, and present the results of the dry measurements. We also describe the calculational methods and models used in the associated analyses, and compare the calculated and measured results.

\section{EXPERIMENTS}

\section{Description of the Narciss M-II Critical_Assembly}

The Narciss M-II critical assembly is a small, zirconium hydride-moderated, epithermal reactor with 37 single-cell, in-core, simulated thermionic fuel elements (TFEs). A stack of five $\mathrm{ZrH}$ moderator blocks forms the primary physical structure of the reactor core. When stacked, the cylindrical blocks form a structure $375-\mathrm{mm}$ high and 
$260-\mathrm{mm}$ in diameter. On either end of the moderator region are 55 -mm-thick beryllium axial-reflector blocks. Thirty-seven channels, which extend the length of the moderator plus reflector blocks, house stainless-steel guide tubes and simulated TFEs. The fuel elements are arranged in concentric circles with a central simulator surrounded by the remaining 36 elements in 3 rings that contain 6,12 , and 18 TFEs, respectively.

Each TFE is fueled by annular uranium-dioxide $\left(\mathrm{UO}_{2}\right)$ pellets that are $96 \%$ enriched in ${ }^{235} \mathrm{U}$. The pellets, which have a central hole diameter of $4.5 \mathrm{~mm}$ and outer diameter of $17.0 \mathrm{~mm}$, are stacked within the cavity of each TFE emitter. (Note that some of the "wet" configurations discussed in a companion paper use two different fuel-pellet geometries.) The total height of the fuel column, although uniform across the core, can vary from 325 to $375 \mathrm{~mm}$. Annular beryllium-oxide $(\mathrm{BeO})$ pellets on either end of the fuel stack provide axial reflection. These reflector pellets have the same outer diameter as the described fuel, but have a smaller, 3.2-mm-diameter central hole. Axial-reflector pellets are added uniformly to either end of the fuel column to form a total column height of $485 \mathrm{~mm}$. The fuel stacks and upper axial-reflector cores are wrapped in aluminum foil to facilitate loading and unloading of the TFE cores.

The simulated emitter, which is the inner tube of a prototypical TFE, forms a cavity into which the fuel and BeO column is placed. The emitter is manufactured from molybdenum and is coated on its outer surface with tungsten. This $0.1-\mathrm{mm}$-thick tungsten layer is $95 \%$ enriched in ${ }^{184} \mathrm{~W}$ to minimize parasitic neutron capture. The emitter, including its coating, has an inner diameter of $18.3 \mathrm{~mm}$ and wall thickness of $1.1 \mathrm{~mm}$. A $0.55-\mathrm{mm}$ inter-electrode gap separates the emitter and collector. The simulated collector is composed of molybdenum and exhibits an inner diameter of $21.6 \mathrm{~mm}$ and wall thickness of $1.4 \mathrm{~mm}$. Each TFE assembly is inserted into a stainless-steel guide tube that has an inner diameter of $24.8 \mathrm{~mm}$ and wall thickness of $0.65 \mathrm{~mm}$.

Radially, a 0.9-mm-thick stainless-steel shell encompasses the Narciss M-II core. This core region is then surrounded by a 73-mm-thick beryllium radial reflector that comprises 12 rotating drums uniformly separated by 12 hourglass-shaped insets. Six of the drums are designated as safety drums and six are control (or compensating) drums. Each of the 68-mm-diameter drums incorporates a $116^{\circ}$ neutron-absorbing arc composed of a mixture of natural $\mathrm{B}_{4} \mathrm{C}$ and $\mathrm{SiC}$. The poison arc-shaped segments are 5-mm thick and extend the length of the drum. Each drum is thinly canned by stainless steel; the beryllium insets have no cladding. The drums are described as turned "in" when the poison is rotated toward the core (at $0^{\circ}$ ) and turned "out" when the poison is away from the core (at $180^{\circ}$ ).

Below the Narciss M-II core are an aluminum lower tube plate and ring, core-support structures, control-drum drive motors, and instrumentation equipment. Above the core are the aluminum upper tube sheet, stainless-steel sealing rings, and TFE caps. Structures beyond the radial reflector include more detectors, and, in the case of a wateror sand-immersed configuration, a 700 -mm-diameter stainless-steel water tank.

The Narciss M-II assembly is an accurate simulation of the Topaz-2 flight reactor. Major differences between the two systems include the lack of $\mathrm{NaK}$ coolant in the critical assembly, the replacement of the coolant-channel tubes of the flight design by a single stainless-steel guide tube, the absence of the upper and lower plena in the critical assembly, and an unspecified difference in the fuel material. Major components such as moderator blocks, control drums, and reflector insets are the same as those used in the flight system.

\section{Review of Experiments Performed}

The first series of Topaz-2 critical experiments was performed at the Narciss M-II facility during November and December of 1992. These dry experiments investigated the effect on reactivity of varying the assembly active-core height and radial-reflector thickness. Of most interest for our analyses are those configurations incorporating the standard reflector thickness of $73 \mathrm{~mm}$ and having active-core heights of 325,345 , and $375 \mathrm{~mm}$, respectively.

For each of these three configurations, Russian researchers determined the rotational position of the six control drums necessary to maintain a delayed critical assembly. In addition, the reactivity worth of a single control drum was measured by comparing it with a well-calibrated reference drum. Other results of this experimental series include the excess reactivity (with all drums "out") of the configuration, the subcriticality of the assembly with all six control drums "in," and the subcriticality with all safety and control drums "in." To determine positive reactivities, the Russian scientists used control-drum calibrations and period measurements. Subcritical reactivities were determined using source-jerk, rod-drop, and noise-analysis techniques. 
During April 1993, a second set of dry experiments was completed at the Narciss M-II facility. These experiments examined the effect of inserting poison rods into the central cavities of the seven centermost TFEs. Because the results of these measurements have not been translated into English, analysis of this series of experiments is not presented here. These cases are important because without the poison, they form the basis of a good dry-to-wet comparison.

The third set of critical experiments, conducted from mid-May to mid-June 1993, examined the effects of water immersion and flooding on the assembly reactivity. These configurations simulated the core becoming immersed and flooded after rejection of its radial reflector. Results of these experiments are presented in a companion paper on the "wet" experiments.

Begun in late June of 1993, the fourth experimental series used a reference assembly design with the radial reflector in place to investigate the effect of radial immersion of the core and of radial immersion combined with core flooding. The Russian scientists started this series with a dry assembly that was identical to one already studied in December 1992. For this dry configuration, they determined the critical configuration, the excess reactivity, controldrum worth, and control- plus safety-drum worth. They also measured the reactivity worth of removing one, two, and three fuel/reflector columns from the core with the drums in varying positions.

The most recent set of Narciss M-II experiments has focused on the reactivity effect of sand-immersion (with and without internal flooding) of the Topaz-2 critical assembly. Results of these experiments are not yet available in an English translation.

\section{CALCULATIONS}

The heterogeneous configuration of the epithermal-spectrum Topaz-2 design poses an analysis challenge to traditional reactor-physics methodologies. Neither Monte Carlo nor discrete ordinates methodology alone provides the capability to characterize accurately all core physics parameters, including excess reactivity, control-drum worths, temperature coefficients, and others. For the purposes of this investigation, however, the most important physics parameter is the value of $\mathbf{k}_{\text {eff }}$ during normal conditions and under the influence of proposed accident scenarios and reactor modifications. For these analyses, we chose Monte Carlo as the primary calculational method.

Monte Carlo neutron-transport methods randomly sample many histories to determine the average behavior of the particles. Because Monte Carlo is a statistical process, an uncertainty is associated with each calculated value. Running a sufficiently large number of neutron histories generally keeps such uncertainties acceptably small. Typically, the U.S. team follows one million particle histories per calculation, leading to a stated relative error of about $0.1 \%$ in the value of $k_{\text {eff. }}$.

The specific Monte Carlo codes used for the calculations are the MCNP code developed at Los Alamos (Briesmeister 1986) and the MCU-2 code developed at the Kurchatov Institute, Moscow (Gomin et al. 1990). MCNP and MCU-2 are three-dimensional, general-purpose, coupled neutron-photon transport codes that can calculate $\mathbf{k}_{\text {eff }}$ eigenvalues for multiplying systems. They can model any geometry that can be described by quadratic surfaces and tori and they provide easy geometry plotting that facilitates debugging. Because of their very powerful geometric capabilities, MCNP and MCU-2 can model the Topaz-2 reactor in great detail.

MCNP is run with a library of pointwise or continuous-energy cross sections based primarily on ENDF/B-Vevaluated data (Kinsy 1979), thereby eliminating inaccuracies associated with multigroup averaging techniques. A similar but somewhat different library is used in MCU-2 (Gomin et al. 1990). For important scattering substances such as zirconium hydride, beryllium, beryllium oxide, and water, we used thermal-scattering $S(\alpha, \beta)$ data that account for chemical-binding and crystalline-structure effects that become important at low neutron energies. MCNP and MCU-2 have been well benchmarked against analytical calculations and critical experiments (Wagner et al. 1992 and Gomin et al. 1990).

\section{Calculational Model Description}

A detailed $360^{\circ}$ MCNP model of the Narciss M-II critical assembly was developed. The core moderator region is represented by a solid cylinder of zirconium hydride with $260-\mathrm{mm}$ diameter and $375-\mathrm{mm}$ height. Located above and below this structure are beryllium axial-reflector plates, each with the same diameter as the moderator block and 
having a height of $55 \mathrm{~mm}$. The total height of the core region is therefore $485 \mathrm{~mm}$. Thirty-seven cylindrical channels penetrate the full length of these core structures. In the model, these channels are precisely located in the same relative positions as those in the actual critical assembly. Similarly, the varying channel diameters are carefully preserved in the model. Explicitly represented in the calculational model are nine countersunk channels of the upper axial-reflector plate.

Several annular regions are required to accurately model the simulated TFE placed within each channel. The emitter, emitter coating, emitter-10-collector gap, collector, collector-to-guide tube gap, guide tube, and guide tubeto-channel gap are all explicitly modeled. Each of these regions is characterized by a different average height. A single annular volume of fuel in each TFE is used to represent the fuel-pellet column. Similarly, above and below each fuel region are zones that represent the beryllium-oxide axial-reflector columns. In the reference configuration, the height of the fuel is $325 \mathrm{~mm}$ and the height of the BeO regions is $80 \mathrm{~mm}$. As necessary, the fuel column and associated $\mathrm{BeO}$ height are modified so that the total height of material within the emitter is $485 \mathrm{~mm}$. The gap between the fuel/ $\mathrm{BeO}$ and the emitter wall is modeled by a separate annular region. Because the sizes of the fuel central cavity and the $\mathrm{BeO}$ central cavity differ, separate void volumes must be used to represent these regions.

Included in the MCNP model of the Topaz-2 critical assembly is a 1-mm-thick stainless-steel reactor vessel that surrounds the core radially. Beyond the vessel region is the 73-mm-thick beryllium radial reflector. Twelve insets and twelve drums are explicitly represented in the model. Gaps are modeled around each inset and drum. The 68-mm-diameter drums are radially canned in a thin stainless-steel shell. The outer 5-mm annulus of each drum is divided into several sectors, one of which simulates the neutron-poison segment. By using translated MCNP surfaces, the $116^{\circ} \mathrm{B}_{4} \mathrm{C} / \mathrm{SiC}$ absorber on each drum can be rotated to any position relative to the core between $0^{\circ}$ and $180^{\circ}$. The differing average heights of the inset pieces, the beryllium drums, and the absorber segments are all individually represented.

Additional structures included in the MCNP model of the critical assembly include relatively thick upper tube sheet and lower tube plates, lower and upper sealing rings, a cap that covers the upper ends of the simulated TFEs, numerous core-support structures, and the surrounding water tank.

Although the MCNP model of the Narciss M-II critical assembly is a very accurate representation of the experimental system, several uncertainties are known to exist in the computer model. For example, in the MCNP calculational models, the density and isotopic composition of each material were represented as accurately as available data allowed. One area of uncertainty is the detailed fuel isotopic composition, which remains proprietary information. Also, although the bulk density of the $\mathrm{ZrH}$ moderator is well known, the specific composition (including hydrogen concentration) of a particular block is not. In addition, the process used to add hydrogen to the block can lead to (unknown) spatial variation of the hydrogen content.

In the model of the core, no attempt is made to account for variations between individual TFE components. Instead, the model uses an average height for each type of component. For example, although the radial dimensions of the emitter are carefully controlled, the simulated emitter heights range from $612.0 \mathrm{~mm}$ to $612.8 \mathrm{~mm}$. Similarly, collector, guide tube, fuel, and axial-reflector column heights in the computational model are represented by average values. The height of each component is chosen to conserve measured mass values. Small dimensional differences between radial-reflector segments are also ignored in the model.

The radial-reflector region is constructed of numerous beryllium pieces, held together by two steel bands that encircle the reactor. The size of gaps that exist between adjacent reflector segments is unknown. For these gaps, the model assumed a size of $3 \mathrm{~mm}$. Note that instrumentation equipment is not included in the MCNP model. Also, the arrangement of reactor support features is estimated from available data.

As translations of Russian documents that detail the critical-assembly description have become available, we have noted three other discrepancies between the assembly and the MCNP model. First, the thickness of the tungsten coating on the emitter is $0.1 \mathrm{~mm}$; the model assumes a value of $0.15 \mathrm{~mm}$. Second, the $0.9-\mathrm{mm}$-thick reactor vessel was represented by a $1-\mathrm{mm}$ shell in the model. A recent sensitivity study showed these two differences to be insignificant. Third, aluminum foil is wrapped around the fuel/reflector columns. This foil is omitted in the calculational model. Experiment measurements indicate that inclusion of the foil increases the reactivity by about $0.10 \$$. 


\section{BESULTS}

This paper presents results for the following dry Narciss M-II experiments:

- Critical configurations and excess reactivities of basic reference assemblies as a function of active-fuel heights ranging from $325 \mathrm{~mm}$ to $375 \mathrm{~mm}$,

- Control-drum worths as a function of active-fuel height, and

- Reactivity worths associated with the removal of up to three central fuel elements in the $325-\mathrm{mm}$ core.

The experimental results reported in this paper are well documented (Ponomarev-Stepnoi et al. 1993, Glushkov et al. 1993a, Glushkov et al. 1993b, and Ponomarev-Stepnoi et al. 1994).

\section{Beference Critical Configurations}

Results of the measurements on the dry reference configurations are summarized in Table 1 . These measurements were performed for active-fuel heights of $325 \mathrm{~mm}, 345 \mathrm{~mm}$, and $375 \mathrm{~mm}$. Column 2 of the table shows the control-drum configuration at delayed critical, and column 5 shows the measured excess reactivity with all control drums in their outermost position $\left(180^{\circ}\right)$. The calculated values of $k_{\text {eff }}$ obtained from MCNP are shown in columns 3 and 6 for the critical configuration and drums-out configuration, respectively, and the corresponding calculated reactivity value (assuming a $\beta_{\text {eff }}$ value of 0.008 ) for the drums-out configuration is shown in column 7 . The one-sigma relative error of the MCNP calculations was typically $0.09 \%$ or $0.11 \$$. Finally, the difference between the calculated and measured values of reactivity (C-M), in dollars, is shown in columns 4 and 8 for the critical and drums-out configurations, respectively.

The data appearing in Table 1 can be used to determine the relationship between reactivity and active-fuel height. This relationship is presented in Figure 1.

TABLE 1. Measured and Calculated Results for Reference Configurations as a function of Fuel Height. Safety Drums are always in their OUT Position. MCNP Relative Errors are typically $0.09 \%$ or $0.11 \$$.

\begin{tabular}{|c|c|c|c|c|c|c|c|}
\hline \multirow[b]{2}{*}{$\begin{array}{c}\text { Core } \\
\text { Height }(\mathrm{mm})\end{array}$} & \multicolumn{3}{|c|}{ Critical Configuration } & \multicolumn{4}{|c|}{ All Drums OUT } \\
\hline & $\begin{array}{l}\text { Control-Drum } \\
\text { Positions }\end{array}$ & $\underset{\mathbf{k}_{\text {eff }}}{\text { MCNP }}$ & $\begin{array}{l}(C-M) \\
\Delta p(\$)\end{array}$ & $\begin{array}{l}\text { Meas'd } \\
\rho(\$)\end{array}$ & $\begin{array}{c}\text { MCNP } \\
\mathbf{k}_{\text {eff }}\end{array}$ & $\begin{array}{l}\text { MCNP } \\
\rho(\$)\end{array}$ & $\begin{array}{l}(\mathrm{C}-\mathrm{M}) \\
\Delta p(\$)\end{array}$ \\
\hline 375 & $\begin{array}{l}\# 2,4,6 \mathrm{IN} \\
\# 5 @ 107^{\circ}\end{array}$ & 0.9871 & -1.63 & 2.35 & 1.0043 & 0.54 & -1.81 \\
\hline 345 & $\begin{array}{c}\# 2 \mathrm{IN} \\
\# 5 @ 55^{\circ}\end{array}$ & 0.9855 & -1.83 & 1.21 & 0.9948 & -0.66 & -1.87 \\
\hline 325 & 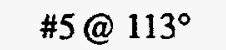 & 0.9858 & -1.80 & 0.15 & 0.9867 & -1.68 & -1.83 \\
\hline
\end{tabular}

\section{Control-Drum Worths}

Of the 12 rotating control drums in the Narciss M-II reflector, 6 are designated as compensating (or control) drums (CD) and 6 as safety drums (SD). The compensating drums and safety drums alternate in a clockwise direction with safety drum \#1 at the 5:30 o'clock position and compensating drum \#1 at the 6:30 o'clock position.

Subcriticality measurements were performed for the following drum configurations:

- Safety drums IN and compensating drums IN, and

- Safety drums OUT and compensating drums IN. 


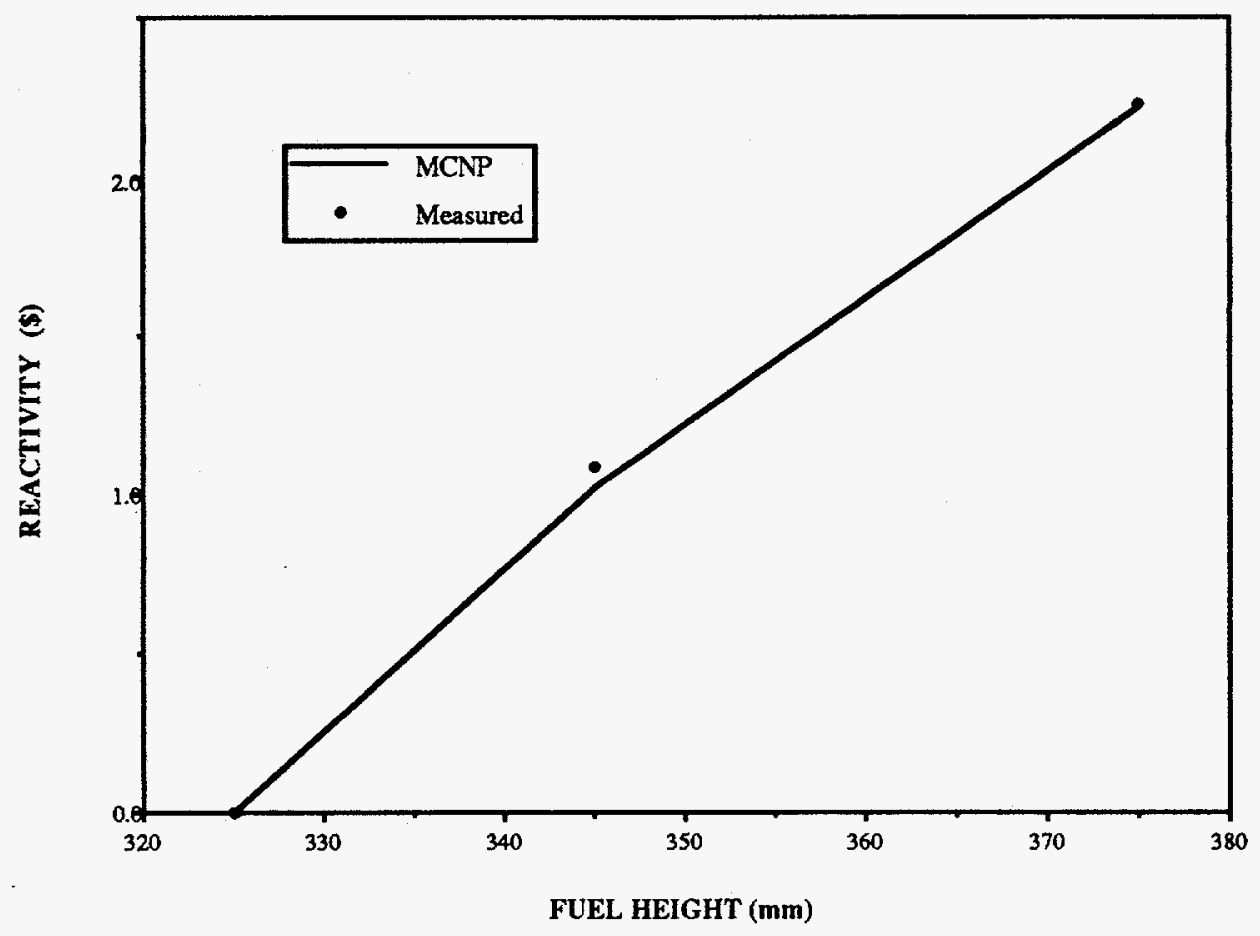

FIGURE 1. Measured and MCNP-Calculated Reactivity Worth as a function of Fuel Height of the Topaz-2 Core.

The measured and MCNP-calculated subcriticality and drum worths are shown in Table 2 for the "all drums IN" configuration and in Table 3 for the "SDs OUT and CDs IN" configuration. Replicate-measurement results are shown for the 325-mm core. The difference between the calculated and measured subcritical reactivities (C-M), in dollars, and the ratio of calculated-to-measured drum worths (C/M) are also shown in the tables.

\section{Reactivity Worth of Central Fuel Elements}

Two anticriticality systems were proposed to keep the reactor subcritical during postulated preorbital accidents: the removal of a selected number of fuel elements from the core, and the insertion of poison rods into selected fuel cavities. The fuel would be reinserted, or the poison would be removed, only after a safe-nuclear orbit was established. The Narciss M-II experimental program included investigations to measure the effectiveness of these modifications. The reactivity effects of fuel removal and poison insertion were measured in both the dry and wet configurations. This report summarizes the effects of removing up to three fuel elements from the central portion of the dry reactor. The experimental results involving insertion of poison into the core are not yet available in an English translation.

Table 4 summarizes the subcritical measurements associated with removing fuel (and axial-reflector) elements from alternate positions in the inner ring of six elements. These measurements were made on the $325-\mathrm{mm}$ core with all the poison drums turned fully out. The listed measured subcritical reactivities include the average of 3 replicate measurements reported. The table shows the differences between the calculated and measured reactivities of the various configurations as well as the reactivity worth of the fuel removed relative to the reference (unperturbed) configuration. Figure 2 provides a graph of reactivity worth as a function number of fuel elements removed from the core of the Narciss M-II assembly.

\section{DISCUSSION}

Reactivity measurements are most accurate when made at or near critical. Examination of the data for the reference configurations made either at critical or with the drums turned out shows that the calculated values are approximately $1.8 \$$ less than the corresponding measured values. This bias remains relatively constant as the fuel height varies 
from $325 \mathrm{~mm}$ to $375 \mathrm{~mm}$, indicating that the change in fuel height is well predicted. The observed bias may be the result of inaccuracies in either the nuclear data used or in the calculational model. The corresponding MCUcalculated bias for the basic configurations is approximately $1.2 \$$ (compared with $-1.8 \$$ for $M C N P$ ).

TABLE 2. Measured and Calculated Control-Drum Worths. All Drums are IN. MCNP Relative Errors are typically $0.09 \%$ or $0.11 \$$.

\begin{tabular}{|c|c|c|c|c|c|c|c|}
\hline \multirow[b]{2}{*}{$\begin{array}{l}\text { Core } \\
\text { Height } \\
(\mathrm{mm})\end{array}$} & \multicolumn{4}{|c|}{ Subcritical Reactivity } & \multicolumn{3}{|c|}{ Drum Worth } \\
\hline & $\begin{array}{l}\text { Meas'd } \\
\rho(\$)\end{array}$ & $\begin{array}{c}\mathrm{MCNP} \\
\mathbf{k}_{\text {eff }}\end{array}$ & $\begin{array}{c}\text { MCNP } \\
\rho(\$)\end{array}$ & $\begin{array}{l}(C-M) \\
\Delta p(\$)\end{array}$ & $\begin{array}{c}\text { Meas'd } \\
\Delta p \text { Drum } \\
(\$)\end{array}$ & $\begin{array}{c}\text { MCNP } \\
\Delta \rho \text { Drum } \\
\text { (\$) }\end{array}$ & $\begin{array}{c}\mathrm{C} / \mathrm{M} \\
\Delta \rho \text { Drum }\end{array}$ \\
\hline 375 & -4.59 & 0.9530 & -6.17 & -1.58 & -6.94 & -6.70 & 0.97 \\
\hline 345 & -5.37 & 0.9459 & -7.15 & -1.78 & -6.58 & -6.49 & 0.99 \\
\hline 325 & -6.12 & 0.9391 & -8.11 & -1.99 & -6.27 & -6.42 & 1.02 \\
\hline$"$ & -6.80 & $"$ & $"$ & -1.31 & -6.95 & $"$ & 0.92 \\
\hline$"$ & -6.2 & $"$ & $"$ & -1.91 & -6.35 & $"$ & 1.01 \\
\hline$"$ & -6.0 & $"$ & $"$ & -2.11 & -6.15 & $"$ & 1.04 \\
\hline
\end{tabular}

TABLE 3. Measured and Calculated Control-Drum Worths. Compensating Drums are IN and Safety Drums are OUT. MCNP Relative Errors are typically $0.09 \%$ or $0.11 \$$.

\begin{tabular}{|c|c|c|c|c|c|c|c|}
\hline \multirow[b]{2}{*}{$\begin{array}{c}\text { Core } \\
\text { Height } \\
(\mathrm{mm})\end{array}$} & \multicolumn{4}{|c|}{ Subcritical Reactivity } & \multicolumn{3}{|c|}{ Drum Worth } \\
\hline & $\begin{array}{l}\text { Meas'd } \\
\rho(\$)\end{array}$ & $\begin{array}{c}\mathrm{MCNP} \\
\mathbf{k}_{\mathrm{eff}}\end{array}$ & $\begin{array}{c}\text { MCNP } \\
\rho(\$)\end{array}$ & $\begin{array}{l}(C-M) \\
\Delta \rho(\$)\end{array}$ & $\begin{array}{c}\text { Meas'd } \\
\Delta \rho \text { Drum } \\
\text { (\$) }\end{array}$ & $\begin{array}{c}\text { MCNP } \\
\Delta \rho \text { Drum } \\
\text { (\$) }\end{array}$ & $\begin{array}{c}\mathrm{C} / \mathrm{M} \\
\Delta \rho \text { Drum }\end{array}$ \\
\hline 375 & -1.93 & 0.9749 & -3.21 & -1.28 & -4.28 & -3.75 & 0.88 \\
\hline 345 & -2.98 & 0.9639 & -4.69 & -1.71 & -4.19 & -4.03 & 0.96 \\
\hline 325 & -4.11 & 0.9582 & -5.46 & -1.35 & -4.26 & -3.77 & 0.89 \\
\hline$"$ & -4.15 & $"$ & $"$ & -1.31 & -4.30 & $"$ & 0.88 \\
\hline$"$ & -4.10 & $"$ & $"$ & -1.36 & -4.25 & $"$ & 0.89 \\
\hline$"$ & -3.91 & $"$ & $"$ & -1.55 & -4.06 & $"$ & 0.93 \\
\hline$"$ & -3.88 & $"$ & $"$ & -1.58 & -4.03 & $"$ & 0.94 \\
\hline$"$ & -3.85 & $"$ & $"$ & -1.61 & -4.00 & $"$ & 0.94 \\
\hline
\end{tabular}


TABLE 4. Worth of Fuel Columns Removed from Inner Ring of 6. Core Height is $325 \mathrm{~mm}$. All Control Drums are OUT. MCNP Relative Errors are typically $0.09 \%$ or 0.11 .

\begin{tabular}{|c|c|c|c|c|c|c|c|}
\hline \multirow[b]{2}{*}{$\begin{array}{c}\text { Fuel } \\
\text { Config. }\end{array}$} & \multicolumn{4}{|c|}{ Critical Configuration } & \multicolumn{3}{|c|}{ Fuel Worth } \\
\hline & $\begin{array}{l}\text { Meas'd } \\
\rho(\$)\end{array}$ & $\begin{array}{c}\text { MCNP } \\
\mathbf{k}_{\text {eff }}\end{array}$ & $\begin{array}{c}\text { MCNP } \\
\rho(\$)\end{array}$ & $\begin{array}{l}(C-M) \\
\Delta p(\$)\end{array}$ & $\begin{array}{c}\text { Meas'd } \\
\Delta p \text { Fuel } \\
\text { (\$) }\end{array}$ & $\begin{array}{c}\text { MCNP } \\
\Delta p \text { Fuel } \\
\text { (\$) }\end{array}$ & $\begin{array}{c}\mathrm{C} / \mathrm{M} \\
\Delta \rho \text { Fuel }\end{array}$ \\
\hline All 37 In & 0.12 & 0.9867 & -1.68 & -1.80 & 0.00 & 0.00 & - \\
\hline \#2 Fuel & -1.45 & 0.9748 & -3.23 & -1.78 & -1.57 & -1.54 & 0.98 \\
\hline \multirow[t]{2}{*}{ Removed } & -1.50 & $"$ & $"$ & -1.73 & -1.62 & $n$ & 0.95 \\
\hline & -1.48 & $n$ & $"$ & -1.75 & -1.60 & $"$ & 0.96 \\
\hline AVERAGE & 1.48 & $"$ & $"$ & -1.75 & -1.60 & $"$ & 0.97 \\
\hline$\# 2, \# 4$ & -3.02 & 0.9634 & -4.75 & -1.73 & -3.14 & -3.07 & 0.98 \\
\hline Fuel & -3.09 & $"$ & $"$ & -1.66 & -3.21 & $"$ & 0.96 \\
\hline Removed & -3.06 & $"$ & $"$ & -1.69 & -3.18 & $"$ & 0.97 \\
\hline AVERAGE & 3.06 & $"$ & $"$ & -1.70 & -3.18 & $"$ & 0.97 \\
\hline$\# 2, \# 4, \# 6$ & -4.51 & 0.9517 & -6.35 & -1.84 & -4.63 & -4.66 & 1.01 \\
\hline Fuel & -4.71 & $"$ & $"$ & -1.64 & -4.83 & $"$ & 0.97 \\
\hline Removed & -4.64 & $"$ & $"$ & -1.71 & -4.76 & $"$ & 0.98 \\
\hline AVERAGE & 4.62 & $"$ & $"$ & -1.73 & -4.74 & $"$ & 0.98 \\
\hline
\end{tabular}

The calculations reported in the previous section were based on ENDF/B-V cross-section evaluations. Recently, ENDF/B-VI cross sections have become available for MCNP. We calculated the $375-\mathrm{mm}$ core with control drums "out" using the ENDF/B-VI cross sections. The reactivity increased by $1.26 \$$ thereby reducing the observed bias to $-0.54 \$$. The primary contributors to the calculated differences between the ENDF/B-V and ENDF/B-VI results were the uranium and steel isotopes.

Control-drum worth experiments were performed to determine the worth a single drum, 6 drums, and 12 drums for the 3 reference cores. Because the worth of a single drum was small relative to the MCNP statistical errors, single drum worths were not included in the analyses. The calculated worths of all 12 drums are in excellent agreement with the measured results. The ratio of the calculated to measured values $(\mathrm{C} / \mathrm{M})$ range from 0.97 for the $375-\mathrm{mm}$ core, to 1.00 for the average of the replicate measurements on the $325-\mathrm{mm}$ core. The replicate measurements on the $325-\mathrm{mm}$ core exhibit a relative standard error of $5.7 \%$. Agreement is not quite as good for the worth of the 6 compensating drums. For these measurements, the $\mathrm{C} / \mathrm{M}$ ratio ranges from 0.88 to 0.96 for the 3 core sizes, while replicate measurements on the $325-\mathrm{mm}$ core exhibit a relative standard error of $3.3 \%$.

The calculated effects of removing fuel elements from the center of the core are in very good agreement with the experimental results. The average $\mathrm{C} / \mathrm{M}$ ratio for the 9 measurements reported in Table 4 is $0.972 \pm 0.018$. The relative error for the 3 series of replicate measurements are $1.6 \%, 1.1 \%$ and $2.2 \%$ for the removal of 1,2 , and 3 fuel 
elements, respectively. The linear relationship of reactivity worth as a function of fuel elements removed is shown graphically in Figure 2.

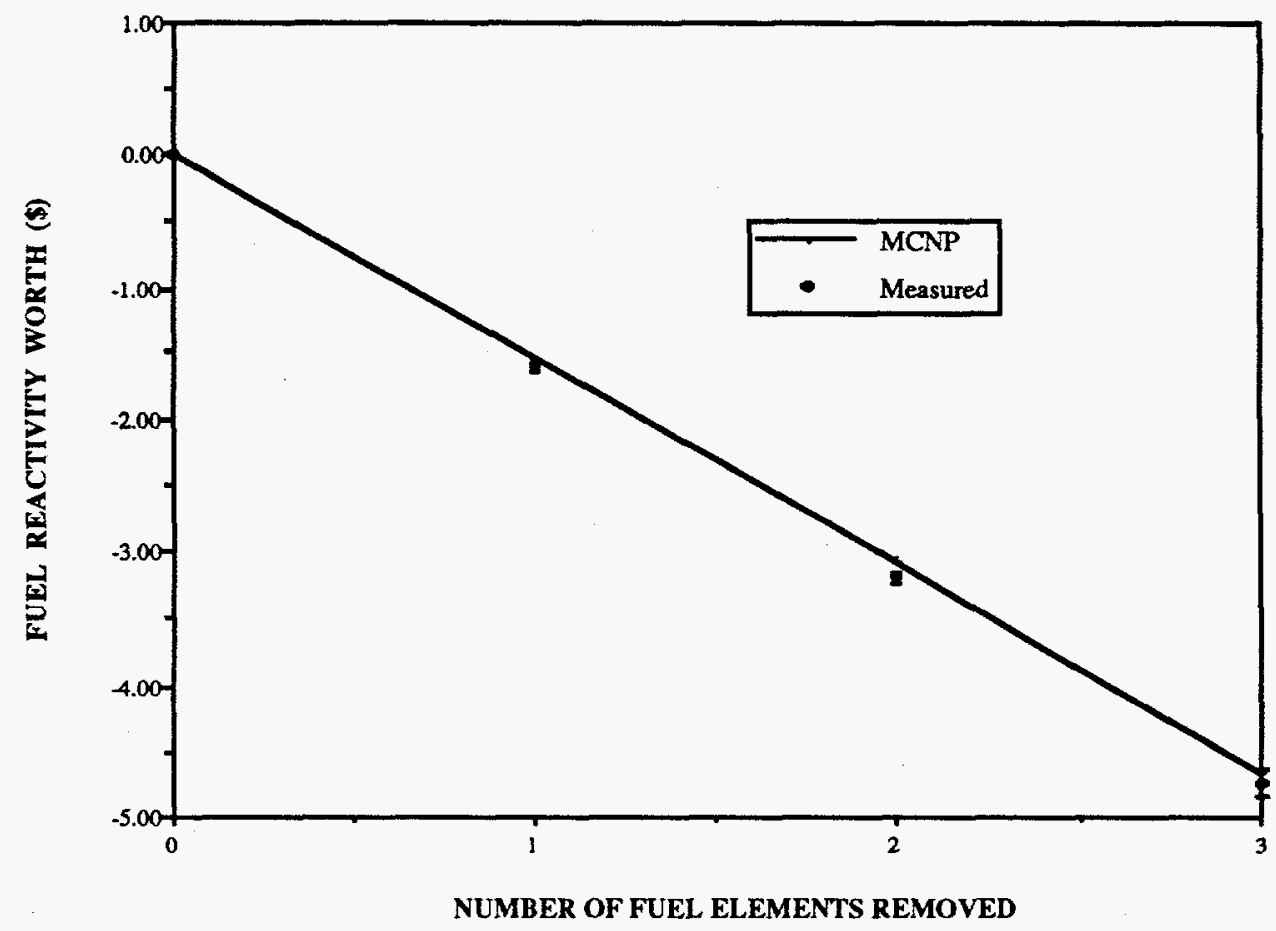

FIGURE 2. Measured and MCNP-Calculated Reactivity Worth as a function of Number of Fuel Elements Removed from the Topaz-2 Core.

\section{SUMMARY AND CONCLUSIONS}

A critical-experiments program was initiated on the Narciss M-II at the Kurchatov Institute to determine the effect of water and sand immersion on the reactivity of the Topaz-2 reactor and to investigate the efficacy of proposed reactor modification to prevent criticality. These experiments also serve as benchmarks to evaluate the methods and data used in the Topaz-2 safety analyses.

Initial dry experiments determined the critical position and excess reactivity of three reference assemblies as a function of fuel height. Additional investigations presented in this paper include the determination of control-drum worths and the reporting of reactivity effects caused by removing up to three fuel elements from the central region of the core.

Both the U.S. and Russian teams used Monte Carlo transport methods to analyze the critical experiments. The specific Monte Carlo codes used for the calculations were the MCNP code developed at Los Alamos and the MCU-2 code developed at the Kurchatov Institute. For the reference dry configurations, the MCNP calculations using ENDF/B-V cross-section evaluations produce a negative reactivity bias of approximately $1.8 \$$. This bias remains constant as the fuel height is varied, so that the effect of changing fuel height is accurately predicted. Similarly, other measured perturbations on the reference configurations such as control-drum worths and fuel-removal worths are also well calculated. The incorporation of more recent ENDF/B-VI cross-section evaluations into the MCNP code reduces the calculated bias from $-1.8 \$$ to $-0.5 \$$. The corresponding bias produced by the MCU- 2 calculations for the reference dry configurations is approximately $1.2 \$$. 


\section{Acknowledgments}

The Russian experimental work described in this paper was funded by the Ballistic Missile Defense Organization under the Department of Defense. The Los Alamos evaluation and analyses were mostly performed with internal funds. The principle authors are employed by the Los Alamos National Laboratory, which is operated by the University of California for the Department of Energy, and by the Kurchatov Institute in Moscow, Russia.

\section{References}

Briesmeister, J. F., Ed. (1986) "MCNP--A General Monte Carlo Code for Neutron and Photon Transport," Version 3A, Los Alamos National Laboratory Report LA-7396-M Rev. 2, Los Alamos, New Mexico.

Glushkov, E. S. et al. (1993a) "Joint Computational Model of Critical Assemblies in Experiments Simulating the TOPAZ-2 Accidental Water Immersion," JV INERTEK Report JV-15/105, Moscow, Russia, August 8, 1993.

Glushkov, E. S. et al. (1993b) "Analysis of TOPAZ-2 Neutronics Under Water Immersion Accidents and Discussion of Possible Options of Reactor Modification to Assure Its Subcriticality," JV INERTEK Report JV-15/178, Moscow, Russia.

Gomin, E. A., L. V. Maiorev, and M. S. Kudkevich (1990) "Some Aspects of Monte-Carlo Method Application to Nuclear Reactor Analysis," Progress in Nuclear Energy, 24: 211-222.

Kinsy, R., Comp. (1979) "ENDF-201: ENDF/B Summary Documentation," Brookhaven National Laboratory Report BNL-NCS-17541 (ENDF-201), 3rd Edition ENDF/B-V, Brookhaven, New York.

Ponomarev-Stepnoi, N. N. et al. (1993) "Performance of Critical Experiments with Variation of the Side Reflector Thickness and the Fuel Element Height," JV INERTEK Report 9349, Moscow, Russia.

Ponomarev-Stepnoi, N. N. et al. (1994) "Experimental Results of Reactivity Effects Research at Narciss M-2 Flooding (Beryllium Reflector On) with Additional Studies of Fuel Removal," JV INERTEK Report JV-15/213, Moscow, Russia, April 28, 1994.

Wagner, J. C., J. E. Sisolak, and G. W. McKinney (1992) "MCNP: Criticality Safety Benchmark Problems," Los Alamos National Laboratory Report LA-12415, Los Alamos, New Mexico.

\section{DISCLAIMER}

This report was prepared as an account of work sponsored by an agency of the United States Government. Neither the United States Government nor any agency thereof, nor any of their employees, makes any warranty, express or implied, or assumes any legal liability or responsibility for the accuracy, completeness, or usefulness of any information, apparatus, product, or process disclosed, or represents that its use would not infringe privately owned rights. Reference herein to any specific commercial product, process, or service by trade name, trademark, manufacturer, or otherwise does not necessarily constitute or imply its endorsement, recommendation, or favoring by the United States Government or any agency thereof. The views and opinions of authors expressed herein do not necessarily state or reflect those of the United States Government or any agency thereof. 